\title{
Violin mode amplitude glitch monitor for the presence of excess noise on the monolithic silica suspensions of GEO 600
}

\author{
B. Sorazu, K. A. Strain, I. S. Heng and R. Kumar \\ Department of Physics and Astronomy, University of Glasgow, University \\ Avenue, Glasgow G12 8QQ, United Kingdom \\ E-mail: b.sorazu@physics.gla.ac.uk
}

\begin{abstract}
Non-Gaussian features of data from gravitational wave detectors are of interest as unpredictable "glitches" limit the sensitivity of searches for many kinds of signal. We consider events due to non-random excitations of the test masses and their suspension fibres. These events could, for example, be related to acoustic emissions in the fibres due to the presence and propagation of cracks or another type of structural perturbation, and they would generate excess noise above the Gaussian background, which matches the level expected due to thermal noise. We look for excess noise in the fundamental violin modes of the monolithic silica suspension fibres of GEO 600. We describe the algorithm used to monitor the violin mode amplitude for glitches, present our results and consider how these may be applied to advanced detectors. The conclusion of our analysis is that no excess noise above what was considered to be thermal noise was observed for several days of $h(t)$ data analysed at the frequency of selected violin modes.
\end{abstract}

PACS numbers: 04.80.Nn, 95.55.Ym, 95.75.Kk, 95.75.Pq

\section{Introduction}

The most sensitive gravitational wave detectors (LIGO [1], VIRGO [2], GEO 600 [3] and TAMA 300 [4]) employ the technique of laser interferometry to compare the shortterm changes in length of two approximately orthogonal "arms". The arms are defined by the light path between mirrors and a beam-splitter all of which are suspended as pendulums and on vibration isolation systems in vacuum to isolate against external sources of vibration. The mirror (and beam-splitter) suspensions are commonly formed by looping one or more steel wires around the fused silica cylindrical optic. In the case of GEO 600, however, fused silica fibres replace the steel wires. The fibres are welded on to suitably shaped "ears" that are bonded one each side to flats polished to form left and right sides on the otherwise cylindrical substrate [5]. The hydroxy-catalysis method is used to form a bond roughly 50 to $100 \mathrm{~nm}$ thick [6]. For technical reasons the tops of the fibres in GEO 600 are supported by ears on an "intermediate mass" the same size as the mirror but made from lower quality fused silica.

Although external sources of vibration can be isolated, thermal noise, which can be viewed in terms of the fluctuation-dissipation theorem as resulting from mechanical loss in the materials [7], cannot be eliminated with components at finite temperature. 
The main reason for choosing fused silica for the fibres in GEO 600 is that it has low dissipation and hence contributes reduced thermal noise. All of the other components in the suspensions contribute thermal noise, including the mirror substrates, the dielectric coatings on the mirrors, the ears and the bonds between ears and mirror.

In GEO 600 it is believed [8] that the noise sources associated with the interferometric measurement of the relative positions of the mirrors (i.e. laser noise, sensing noise, oscillator noise, etc.) exceeds and thus obscures thermal noise over most of the observing band, which extends from $50 \mathrm{~Hz}$ to about $3 \mathrm{kHz}$. An exception is in the close neighbourhood of what we term the "violin modes". These are the transverse modes of vibration of the suspension fibres oscillating between the almost-rigid constraints represented by the mirror and intermediate mass. We could approximate these oscillating thin suspension fibres under considerable stress as oscillators which are in a state of thermal equilibrium with the environment. The motion of these oscillators induced by thermal fluctuations (thermal noise) is Brownian in nature and results in oscillations with random amplitude and phase in every mode of oscillation [9]. In particular the component of the vibration along the sensitive axis of the interferometer leads to motion of the mass that is sensed by the interferometry. This motion is of reduced amplitude, however, as the test mass of many kg reacts to a lesser degree. The randomness associated to the Brownian motion of the interferometric detectors test masses due to thermal noise, shows as changes in the amplitude of the violin modes with a normal (or Gaussian) distribution.

The focus of the current work is to search for events which lead to excitation of the violin modes such that their amplitude variation with time is inconsistent with thermal noise and therefore does not have a Gaussian distribution.

This is of interest because such events, evidenced in the form of unexpected "glitches" $\ddagger$, limit the sensitivity of searches for several classes of gravitational wave signal. Non-Gaussian events of this type are known to occur in steel suspension metal wires due to stress-release processes [10, 11, 12, 13]. Similar stress-release processes may happen in some of GEO 600 suspension components, such as fibres and bonds which are permanently under stress. Additionally as the "next generation" detectors, Advanced LIGO [14, 15] and Advanced Virgo [16], are planned to implement fused silica suspension fibres it is of interest to consider whether information can be extracted from the experience of using similar technology in GEO 600.

We are aware of previous work done on a test setup of fused silica fibres alone under tension to observe any non-thermal excitation $[17,18]$. Their conclusion is that no evidence of non-thermal excitation was observed. However their measurements only applied to silica fibres on a down-scaled model of a suspension, meanwhile the work presented here concentrates on a monolithic fused silica suspension for a full scale ground based gravitational wave interferometric detector in long term operation.

This paper is structured as follows; first we establish that the observed typical

$\ddagger$ A glitch is defined, in the context of this text, as a transient signal within the detector recorded output. These transients could be due to gravitation wave sources (short duration violent astronomical events) or most probably to noise transients of instrumental and/or environmental origin due to the great sensitivity of current interferometric gravitational wave detectors. 
amplitude of vibration of the mirrors in GEO 600 at the fundamental violin mode resonances, of at least some of the suspension fibres, corresponds to the expected thermal energy excitation. This is achieved with the help of a detailed and accurate model of GEO 600 suspensions using a Finite Element Method (FEM). The FE model provides the relationship between thermal energy, apparent thermal force, and vibration of fibres and mirrors, which were compared to the vibration of the mirrors observed in GEO 600 at the fundamental violin mode frequency. We follow with the description of a computer algorithm designed to monitor the violin mode amplitude for glitches. This algorithm is later applied to several violin modes which are analyzed over timescales of the order of 1 day to look for non-Gaussian events. The result is expressed in terms of the rate, or an upper limit on the rate, of events of a given force. We finish this paper by considering how these results may be applied to advanced detectors.

\section{Finite elements model of GEO 600 suspension}

The main objective of this paper is to look for non-Gaussian events affecting GEO 600 suspensions by detecting a non-Gaussian distribution on the changes in the amplitude of the violin modes. However this only would be true if the observed violin mode amplitude is consistent with thermal noise. This would also allow us to extend the results observed in GEO 600 to other systems based in similar suspensions.

In principle we could have taken an analytical approach to show that the observed violin mode amplitude was dominated by an excitation equivalent to the expected thermal energy. However due to the need to represent fibres as relatively complex tapered beams, a finite element approach was favourable. The finite element model approximated a GEO 600 test mass (mirror of weight $5.6 \mathrm{~kg}$ ) and its four supporting tapered fibres (with length of $28 \mathrm{~cm}, 10 \mathrm{~mm}$ apart, $300 \mu \mathrm{m}$ at the thinnest section and $3 \mathrm{~mm}$ at the stock ends). Fibres of slightly different properties were simulated as no two fibres in GEO 600 are identical and this allowed to break the degeneracy between the fibres in the model to allow the individual outcomes to be extracted. Tapered and un-tapered (uniform cross section of $300 \mu \mathrm{m}$ ) fibres were used for comparison as well as one fibre with a $10 \%$ change in its density (as shown on the FE model diagram of GEO 600 suspension shown in figure 1).

The FE model was firstly solved under a static analysis which provided the equilibrium state of the model under gravity which was later used as the initial state for a modal analysis. The modal analysis provided all natural frequencies of the system. The fundamental violin mode of one fibre was investigated. The energy of motion and displacement of the anti-node of the mode (close to the middle of the fibre) and of the mass were compared. This allowed the amplitude of the violin mode, when excited with the expected thermal energy, to be estimated.

The GEO 600 suspension has a high mechanical quality factor (Q). To reduce the settling time after disturbance the fibres were damped by application of polytetrafluoroethane on carefully chosen segments of the total length [19]. This reduces the $\mathrm{Q}$ of the first few modes to $\sim 10^{7}$. The fundamental violin mode frequencies in GEO 600 are around $700 \mathrm{~Hz}$, and so a long measurement is needed to resolve the width of resonance. This is not attempted in the current work. Integrating over the narrow 


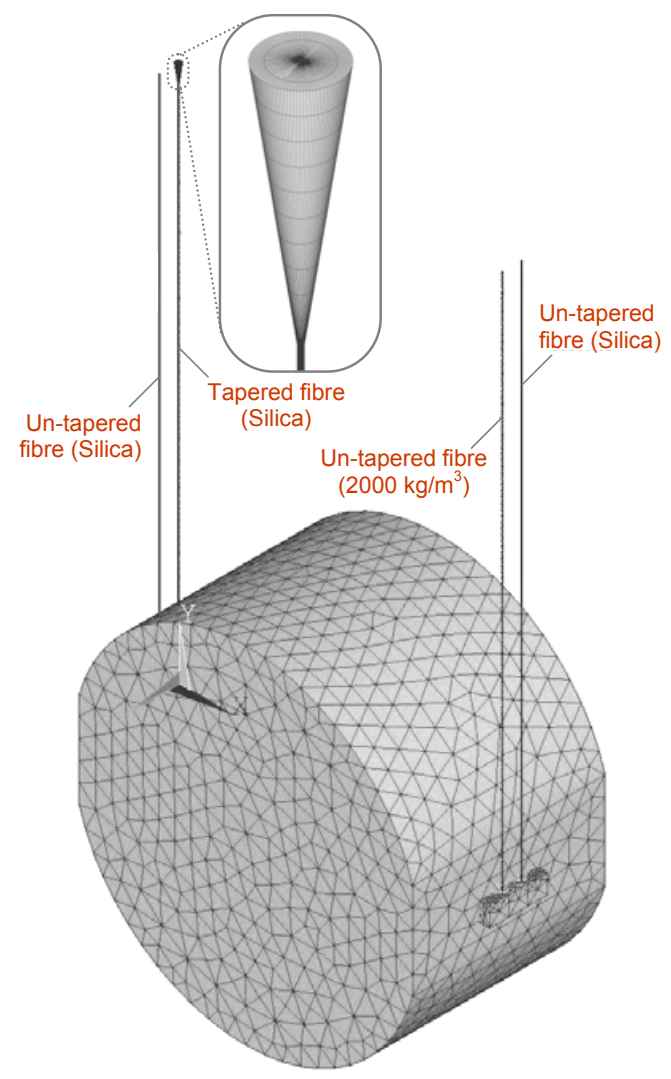

Figure 1. FE model of GEO 600 test mass and the simulated 4 types of supporting fibres. A zoom of the modeled tapered region is also displayed.

resonance, the rms thermal noise level is independent of the $\mathrm{Q}$, as it arises from the usual mode energy of $k_{b} T / 2$ per degree of freedom (here $k_{b}$ is Boltzmann's constant and $T$ is the temperature). Considering that, in first order, only the longitudinal mode is sensed by the detector then a single degree of freedom is used in the estimation of the expected thermal energy $\left(k_{b} T / 2\right)$. We calibrated the displacement and energy results from the FE model by first relating the computed kinetic energy of each mode with the computed displacement of the $5.6 \mathrm{~kg}$ test mass. Finally by comparing the expected thermal energy to the computed kinetic energy of the mode it was possible to calculate the thermal noise displacement values shown in Table 1.

Table 1 shows the outcome of the simulations for the four different fibres in the model. The values given by the modal analysis of energy and displacement are relative numbers (the modal analysis includes an arbitrary excitation, but returns results in SI units, which allow scaling to the thermal noise excitation). The total energy refers to the energy of the combined suspension (mass and fibre) and system displacement refers to the antinode of the fundamental violin mode of the fibre oscillation. The total energy of system and mass are given as their kinetic energy at the equilibrium position where the elastic potential energy is minimum (or null). The last column in 
Table 1. FE model of GEO 600 suspension analysis results. All columns apart from "Violin mode amplitude $r m s$ " are relative numbers from a modal analysis of the model. $E_{k}$ is the kinetic energy given at the equilibrium position where the elastic potential energy is null.

\begin{tabular}{lccccc}
\hline Fibre design & $\begin{array}{c}\text { Mode } \\
\text { frequency } \\
(\mathrm{Hz})\end{array}$ & $\begin{array}{c}\text { System } \\
\text { displacement } \\
(\mathrm{m})\end{array}$ & $\begin{array}{c}\text { Total } E_{k} \\
(\mathrm{~J})\end{array}$ & $\begin{array}{c}\text { Mass } E_{k} \\
(\mathrm{~J})\end{array}$ & $\begin{array}{c}\text { Violin mode } \\
\text { amplitude } \\
\mathrm{rms}(\mathrm{m})\end{array}$ \\
\hline Un-tapered 1 & 718.5 & 292.9 & $1.02 \cdot 10^{7}$ & 8.68 & $5.6 \cdot 10^{-18}$ \\
\hline Un-tapered 2 & 730.7 & 292.9 & $1.05 \cdot 10^{7}$ & 9.0 & $5.5 \cdot 10^{-18}$ \\
\hline $\begin{array}{l}\text { Un-tapered } \\
\left.\text { (density 2000 } \mathrm{kg} / \mathrm{m}^{3}\right)\end{array}$ & 753.9 & 307.4 & $1.12 \cdot 10^{7}$ & 8.68 & $5.05 \cdot 10^{-18}$ \\
\hline Tapered fibre & 783.7 & 303.4 & $1.21 \cdot 10^{7}$ & 10.03 & $5.06 \cdot 10^{-18}$ \\
\hline
\end{tabular}

table 1, which represents the violin mode amplitude rms (maximum displacement of the mass in the longitudinal direction at the fundamental resonance of the suspension fibres), is already calibrated.

Notice that the violin modes frequencies obtained from the FE model of the different fibres are a few percent higher than the experimental ones (which range from $627 \mathrm{~Hz}$ up to $713 \mathrm{~Hz}$ ). This discrepancy is due to a lack of precise knowledge of the GEO 600 fibres diameter, which range between 210 and $300 \mu$ m depending on the suspension, as well as the simulation fibres not including the damping material applied to GEO 600 fibres. Table 1 shows a spread of no more than $10 \%$ in the values of the violin mode amplitude for the different types of simulated fibres.

Finally we need to calculate the experimental violin mode amplitudes as given by the amplitude spectral density of the gravitational wave stream (called $h(t)$ ). The typical amplitude, after settling, of a fundamental violin mode in GEO 600 is seen as a strain value which could oscillate by a factor of 2 or more depending on the time and violin mode being measured. For instance for the violin mode at $684.9 \mathrm{~Hz}$ the violin mode amplitude lies in the range $7 \cdot 10^{-20}$ to $12 \cdot 10^{-20} / \sqrt{\mathrm{Hz}} \mathrm{rms}$ (for a window bandwidth (FWHM) of $0.012 \mathrm{~Hz}$ ) (see figure 2 as an example, which is the amplitude spectral density of 30 minutes of $h(t)$ GEO 600 data around the violin mode frequency of $684.9 \mathrm{~Hz}$ ). This violin mode is associated to the far mirror in the east arm of the detector (identified as MFe) [6], which contributes with an effective length of $600 \mathrm{~m}$ for the conversion from strain into displacement. Therefore the amplitude of this violin mode $\mathrm{rms}$ oscillates between $4.5 \cdot 10^{-18}$ and $7.9 \cdot 10^{-18} \mathrm{~m}$. The same effective length of $600 \mathrm{~m}$ is contributed by the beam-splitter although the violin modes associated to the central building mirrors would contribute with an effective length of $1200 \mathrm{~m}$.

The values of the violin mode amplitude given by the FE model are within the experimental range of values and so, given any evidence to the contrary, it is reasonable to assume that the observed violin mode amplitude in GEO 600 is consistent with thermal noise.

Therefore any sufficiently large glitch originating as excitation of the suspension fibres, should appear in $h(t)$ as a glitch in the amplitude of the associated violin mode(s). In the following sections we describe the algorithm used to monitor the 


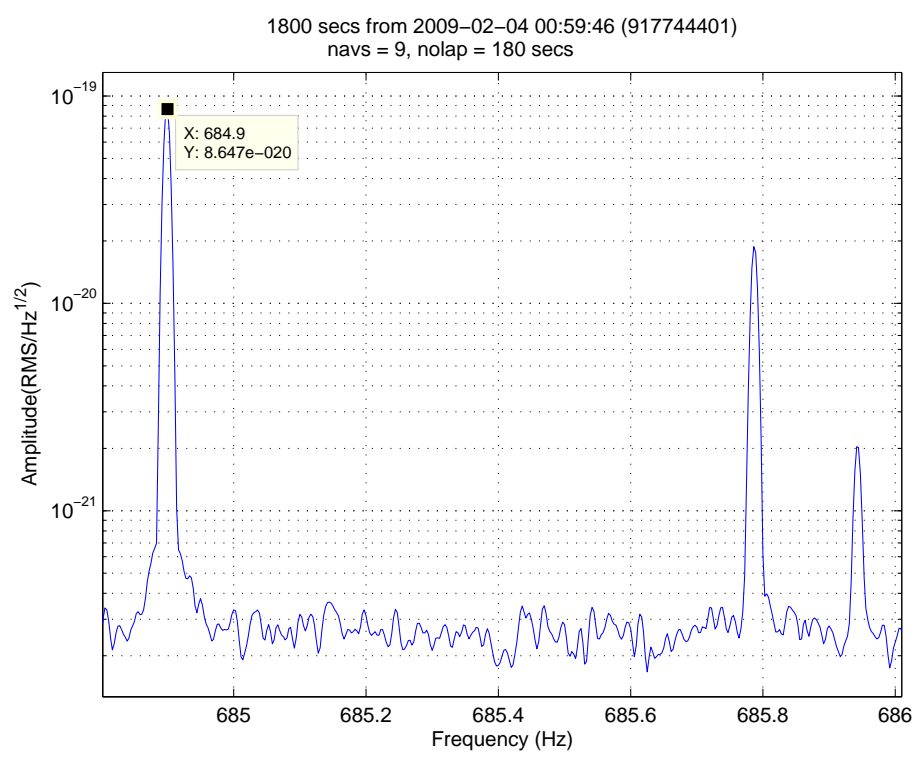

Figure 2. ASD of 30 minutes of $h(t)$ data, chosen at the centre of the 22 hours of the $h(t)$ data used for the analysis in this paper.

violin mode amplitude for glitches, present our results, and consider how these may be applied to advanced detectors.

\section{Algorithm to monitor violin mode amplitude and detect glitches}

The objective of the algorithm is to monitor and record short-term perturbations in the amplitude of the selected violin modes. The block diagram in figure 3 shows its basic principle of operation. The approach employs a heterodyning, filtering and down-sampling procedure followed by differencing to reveal changes in the complex amplitude of the mode.

The input data to the algorithm is calibrated $h(t)$ data. A check is done that that the detector is operating properly and producing valid, calibrated "science" data throughout the period of time to be analysed.

The $h(t)$ data is queried and divided into overlapping segments of equal length (we name these "heterodyning segments"). In each heterodyning segment the frequency of the violin mode is considered constant. In the analysis presented here the data segment duration was typically chosen to be 5 minutes.

The heterodyning segments overlap in order to avoid the discontinuities being generated at the beginning and end of the data segments due to settling in the low pass filtering stages, without losing data. The overlap was taken to be $80 \%$ in the current work. 


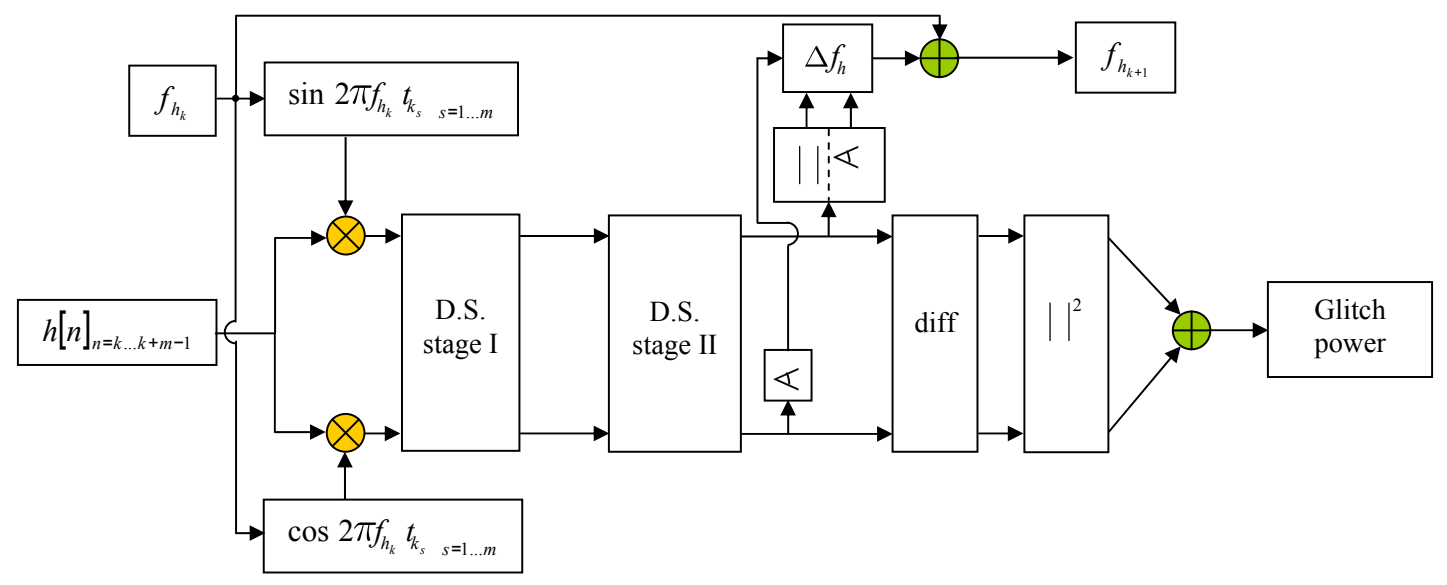

Figure 3. Block diagram of the violin mode amplitude monitor algorithm. The symbols and components in the block diagram are defined as follows; || magnitude, $\varangle$ phase, $\otimes$ multiplication, $\oplus$ addition, D.S. Stage $\mathrm{I}=$ down sample stage I, composed of a IIR (infinite impulse response filter) and posterior down sample, D.S. Stage II = down sample stage II, composed of a FIR (finite impulse response filter) and down sample, $f_{h_{k}}=$ heterodyning frequency of the current iteration (the value used for the first iteration is obtained from a high resolution FFT of the $h(t)$ data used in the analysis), $\Delta f_{h}=$ change in the heterodyne frequency, including both sign and magnitude, diff $=$ differential value or equivalently $x[k+1]-x[k]$.

The monitor requires an initial estimate of the frequency of the violin mode $\left(f_{h_{0}}\right)$. This value can be obtained by plotting the spectrum of, for example, 30 minutes of $h(t)$ data at the centre of the time interval being analysed.

\subsection{Heterodyning}

Each segment of $h(t)$ data is heterodyned and both quadratures are generated by multiplying it by a sine and cosine wave at the appropriate heterodyning frequency (initially $f_{h_{0}}$ ). This brings the mode frequency and any nearby contaminating spectral features in the data to low frequency, where it is easy to pick out the desired component by low-pass filtering. Both quadratures are needed as the initial phase is unknown and the frequency estimate is in any case imperfect.

\subsection{Low pass filtering}

The next stage in the algorithm is low-pass filtering to isolate the violin mode of interest from the adjacent spectrum peaks. As shown in figure 3, this is done by two down sampling stages. The more selective filter is of finite impulse response (FIR), 
an equiripple type due to its suitability to specify a minimum stop-band attenuation. The FIR filter is preceded by a down-sampling stage of a $4^{\text {th }}$ order Butterworth infinite impulse response (IIR) made from two second-order stages to reduce sensitivity to coefficient errors.

Based on an estimate of the required suppression of beats with nearby lines, the FIR filter was chosen to give a minimum stop-band attenuation of $50 \mathrm{~dB}$. A bandpass ripple of $1 \mathrm{~dB}$ was allowed as it was considered not to introduce any noticeable perturbations of the amplitude of the violin mode. The FIR filter also required a narrow transition of around $0.1 \mathrm{~Hz}$. Both the IIR and FIR filters had to be adapted for the different violin modes chosen.

\subsection{Frequency tracker}

It was noticed that over a period of several hours the violin mode frequency would shift gradually by a few tens of $\mathrm{mHz}$, most likely due to temperature drifts of the suspension fibres and drift of the pitch and roll alignment [5]. The former due to a temperature dependency in the Young's modulus of the fused silica and the later due to the temperature dependency of the Young's modulus of the cantilever springs which support the suspension fibres as part of the seismic isolation scheme. As a consequence our algorithm was made to adjust the heterodyning frequency to keep the heterodyne signal near DC. In order to do this, we took into account that the frequency shift $\left(\omega_{h}-\omega_{i}\right)$, in magnitude and sign, of the violin mode is encoded in the sine and cosine heterodyne projections $\left(\sin _{-} h(t)\right.$ and $\cos _{-} h(t)$ respectively):

$$
\text { cos_h }(t)+i \sin \_h(t)=\frac{-A}{2 i} e^{i t\left(\omega_{h}-\omega_{i}\right)}
$$

The absolute value of the Fourier transform of equation 1 would be a Dirac delta at the frequency $\left(\omega_{h}-\omega_{i}\right)$, which is what the frequency tracker should monitor.

\subsection{Glitch power values}

The final part of the violin mode amplitude monitor, represented by the last three blocks in figure 3, combines the sine and cosine heterodyne projections and converts that information into the "glitch power" of the violin mode. First we difference the sine and cosine heterodyne projections individually and then find the square of the absolute value of each output. These are summed to obtain the glitch power.

The input $h(t)$ data to the algorithm is given in strain units, so the output glitch power is given in strain squared units $(\Delta L / L)^{2}$. When considering individual suspensions a displacement is more useful so the output glitch power was converted into glitch displacement squared values by multiplying by the square of the effective arm length of the suspended mass associated to the violin mode being analysed. This effective arm length is of 600 meters for the beam-splitter and far mirrors (these mirrors are identified as MFe (east arm) and MFn (north arm) in GEO 600 and they are the elbow mirrors of GEO 600 folded arms) and of 1200 meters for the arm end mirrors (identified as MCe and MCn in GEO600 because they are situated in the central building together with the beam-splitter). In the glitch power plots of figures 4 and 5 
the length factor was always 600 meters because the corresponding violin modes were associated to the far mirror MFe and the beam-splitter respectively.

This algorithm has been successfully applied to several violin modes over a few days of $h(t)$ data. Next we present the result of some of these analyses.

\section{Results and analysis}

As with any gravitational wave detector there are glitches of various origin. While the violin mode monitor, through its narrow band response, provides good rejection of background glitches - i.e. those that show up other than just at the violin mode frequency, there is some leakage. In testing the algorithm it was observed that selection of only the quietest stretches of data as judged by other means was a necessary starting point. There is no reason to believe that this selection would lead to bias, provided quite long stretches of data were used (and there has been no further selection after the violin mode analysis).

\subsection{Selection of the $h(t)$ data and violin modes to be analyzed}

The selection of adequate $h(t)$ data segments was done by inspection of the time central frequency plots of the glitches detected by HACR $\S$, this is part of the routine data quality monitoring provided for GEO 600 data. The selected data segments were those with the lowest glitch rates and few obvious structures within the glitch concentration, particularly for glitches likely to have significant power around the violin mode frequency. Unfortunately the presence of the violin modes reduces the sensitivity of HACR in the critical band, and so the safe approach was to look for data showing a relative absence of glitches above and below the violin mode band.

GEO 600 provides a data quality channel which gives real time information of the unlocked state of the detector as well as hardware and software maintenance being carried out. We looked for a segment of continuous data (to simplify the analysis) with the detector locked during the entire segment, without any type of maintenance being performed and with low glitch density.

Not all the violin modes are suitable for this analysis. Some violin modes are associated to suspended masses with feedback control forces applied to the masses continuously. Control forces are applied during a re-lock of the interferometer to bring it to its operating point (these masses are the central building mirrors; MCe and $\mathrm{MCn}$ ) and once locked the control forces are applied to keep it locked. These feedback forces would excite the respective violin modes amplitude and any glitches in the control forces would be difficult to distinguish from glitches in the suspension.

$\S$ Hierarchical Algorithm for Curves and Ridges, it is the event trigger generator used in GEO600 to detect transient-burst events [20, 21]. HACR produces glitches when an excess power of short time duration is detected. In order to look for excess powers, HACR divides the gravitational wave signal $h(t)$ into highly overlapped milliseconds segments. Every few seconds a spectrogram is calculated with $32 \mathrm{~Hz}$ frequency resolution and $7.8 \mathrm{msec}$. time resolution. Each of these time-frequency resolution squares is called "pixel". HACR groups those adjacent pixels which are statistically different from the background noise into a single glitch. 
That is why these violin modes are not used in this analysis. The violin modes that do not change in amplitude during an interferometer re-lock are the ones we name stable and they are clearly visible by looking into ASD (amplitude spectral density) plots of $h(t)$ data before and after a re-lock. The stable modes, from which we selected those to be analysed, were observed to be of relatively stable amplitude and show no sign of a step change during detector re-lock. These modes are associated to the fibres for the beam-splitter and the far mirror suspensions; MTe and MTn.

In GEO there are 12 fibres - 4 on each of the two far mirrors and the beam-splitter that are of interest for this work. From these modes we selected a few to analyse based on the nearest neighbour being sufficiently far away in frequency to allow its elimination in the second low-pass filtering stage. It had previously been observed that in some cases the fibres in GEO 600 were of sufficiently non-circular cross section so that their associated violin mode was split in two. Based on this, it is possible that some of the modes selected are actually unresolved pairs, but this should not affect the analysis.

\subsection{Glitch power histograms of stable violin modes}

Putting all the above considerations together 4 violin modes were selected for further analysis at frequencies of $684.9 \mathrm{~Hz}, 688.725 \mathrm{~Hz}, 700.425 \mathrm{~Hz}$ and $713.77 \mathrm{~Hz}$. There was a gap of at least $0.35 \mathrm{~Hz}$ between any mode and its nearest neighbour. The modes were analysed in order of decreasing frequency gap, as it was not clear what the limit would be. For every violin mode being analyzed it was therefore required to readjust the cut-off frequency value of the IIR low pass filter and the stop-band frequency value of the FIR low pass filter in the monitor algorithm, in order to filter out those nearby peaks correctly. The initial heterodyning frequency, overlap between adjacent $h(t)$ data segments and data segment length were also adjusted. Because similar results were obtained for the 4 analysed violin modes, here we only show two sets of results for the modes at $684.9 \mathrm{~Hz}$ and $713.77 \mathrm{~Hz}$ applied over 22 hours of $h(t)$ data. As an example, figure 2 shows the spectrum around the violin mode frequency at $684.9 \mathrm{~Hz}$, obtained from 30 minutes of data from the data segment analysed. The nearby peak is in this case at a distance of $0.9 \mathrm{~Hz}$. Note that the background noise is relatively white, as it was for all but one of the violin modes investigated.

The results for the mode at $684.9 \mathrm{~Hz}$ over 22 hours of continuous $h(t)$ data are shown in figure 4 . The top plot is the normalized histogram and the bottom plot is the normalized cumulative histogram (calculated as the number of glitch power counts above the glitch power threshold given in the horizontal axis) of the glitch power values obtained every 0.875 seconds. The vertical axes are normalized by dividing by the length of the data segment in seconds before taking the logarithm. Standard error bars are given and a straight line was fit to the data. The fitting parameters shown include the "goodness of fit" $R$, and the value very close to unity (0.99996) indicates that the linear fit is valid and so the data follow a Gaussian distribution as expected if the source of glitches is thermal noise. In the cumulative histogram no deviation from a Gaussian distribution is seen to exceed the error bars, therefore no excess noise was observed that would correspond to non-Gaussian glitches. In the normalized histogram we notice a slight deviation from the linear fit at the highest glitch power values, but this occurs because it corresponds to single glitches. For this 
mode the cut-off frequency of the IIR filter was chosen to be $0.4 \mathrm{~Hz}$, which was also the pass-band frequency of the FIR filter with a stop-band frequency of $0.55 \mathrm{~Hz}$. The equivalent noise bandwidth (ENBW) of the combined filters was $0.343 \mathrm{~Hz}$.

Figure 5 shows the normalized histogram and cumulative histogram, of the glitch power values obtained every 1.17 seconds, for the violin mode at $713.77 \mathrm{~Hz}$. For this mode the cut-off frequency of the IIR filter was chosen to be $0.3 \mathrm{~Hz}$, which was also the pass-band frequency of the FIR filter with a stop-band frequency of $0.415 \mathrm{~Hz}$. The ENBW of the combined filters was $0.272 \mathrm{~Hz}$.

Similar results were observed at different times and for all but one of the violin modes. The mode at $700.425 \mathrm{~Hz}$ showed structured background noise of unknown origin (but possibly related to the mains harmonic at 14 times $50 \mathrm{~Hz}$ ). This resulted in quite strong correlations showing up in the glitch distribution. As the effect was seen only in the case of this particular mode, with the excess background noise, we chose to neglect the data.

\section{Applying the results to advanced interferometers}

A consequence of the presence of multiple nearby peaks around each violin mode, is that the bandwidth for monitoring each mode is limited. As the glitch is expected to be of short-duration, the optimum measurement bandwidth, which would be of the order of the glitch bandwidth or the inverse of the glitch duration [22], is much larger than the available measurement bandwidth. The bandwidth of the algorithm is given by the ENBW of the combined filters applied to each violin mode, which as shown above are very narrow with respect to broadband glitches. Therefore the signal recovered is expected to be almost exactly proportional to the measurement bandwidth.

Before the glitch power histograms, obtained here, can be applied to other detectors, such as Advanced LIGO and Advanced VIRGO, we first need to calibrate them in respect to the sensitivity of the algorithm to the glitches. One way of calibrating the glitch values of the analysis is to compare them with the noise amplitude spectral density target for a detector (sensitivity curves). In this case there would be a factor of the square root of the ENBW of combined filters entering any comparison of glitch size with the noise amplitude spectral density target for a detector. The calibration and application of the results here presented is not the objective of this paper, but both are identified as further work.

Nevertheless, as GEO 600 and advanced detectors share the same silica suspension technology, we make the reasonable assumption that glitches associated with these suspensions will be of broadly similar pulse-shape and duration. Thus the conclusions of the current analysis is applicable to advanced detectors, i.e. that no deviation of glitch distribution for what is expected to be thermal noise has been observed in several days of $\mathrm{h}(\mathrm{t})$ data analysed. The glitches might not be identical for GEO 600 and advanced detectors, however, as in the advanced detectors the suspended mirrors are several times heavier. This means that the response to a given (glitch) force is less, but the increased tension in the fibres makes them stiffer, possibly leading to larger initial glitches. Other factors may also influence the difference in the glitches. 

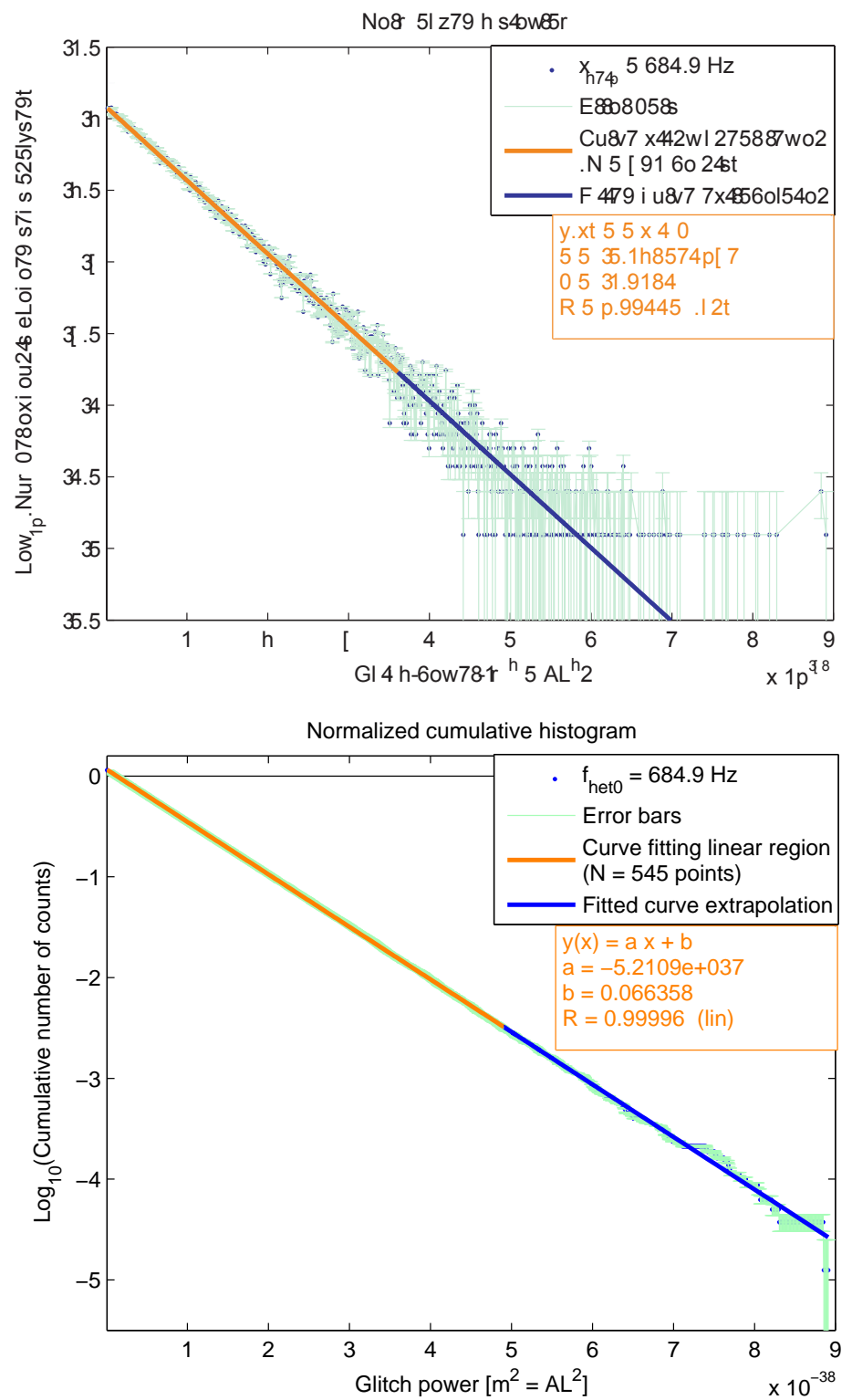

Figure 4. Violin mode at $684.9 \mathrm{~Hz}$ with 22 hours of continuous $h(t)$ data analysed: (top) Normalized histogram of the glitch power values with error bars. (bottom) Normalized cumulative histogram (calculated as the number of glitch power counts above the glitch power threshold given in the horizontal axis) of the glitch power values with standard error bars. No excess of glitches is noticeable.

\section{Conclusions}

The good linear fits of the logarithmic normalized histograms of the power values associated to the glitches on the amplitude of the stable violin modes analysed indicates 

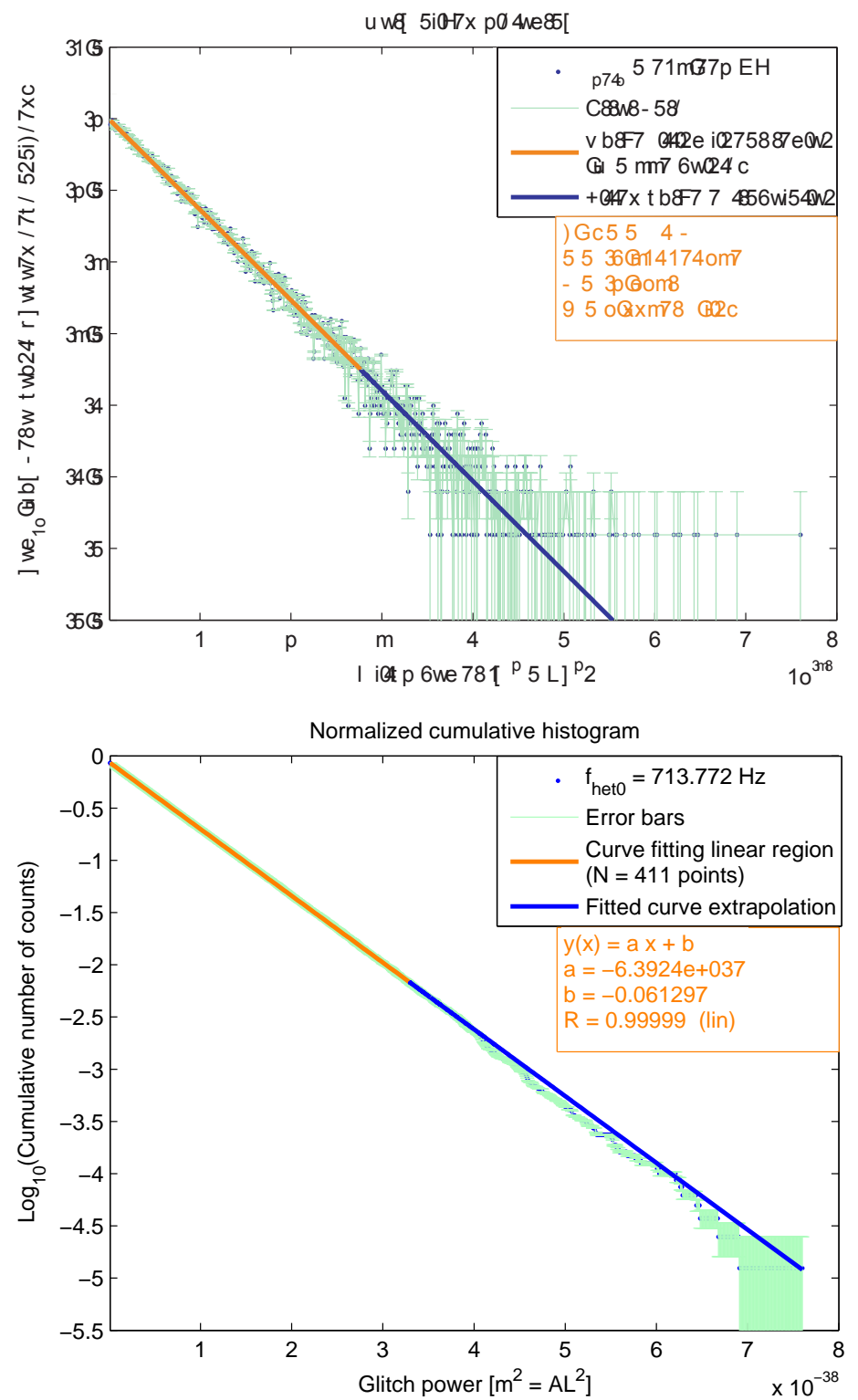

Figure 5. Violin mode at $713.77 \mathrm{~Hz}$ with 22 hours of continuous $h(t)$ data analysed $(t o p)$ Normalized histogram of the glitch power values with error bars. (bottom) Normalized cumulative histogram (calculated as the number of glitch power counts above the glitch power threshold given in the horizontal axis) of the glitch power values with standard error bars. No excess of glitches is noticeable.

a Gaussian distribution as expected if the source of glitches is thermal noise. Because no deviation from a Gaussian distribution is seen to exceed the error bars, no excess noise is observed that would correspond to non-Gaussian glitches, at least at a rate of 1 glitch per day. 
Further work is planned to use the violin mode amplitude algorithm as a realtime monitor of the state of the fused silica suspensions in the way of a potential data quality flag or veto channel.

\section{References}

[1] D. Sigg et al, Status of the LIGO detectors, Class. Quantum Grav. 23, pp. S51-S56, 2006.

[2] F. Acernese et al, The status of VIRGO, Class. Quantum Grav. 23, pp. S63-S69, 2006.

[3] H. Lück et al, The GEO 600 status, Class. Quantum Grav. 27, 084003 (9pp), 2010.

[4] R. Takahashi et al, Status of TAMA 300, Class. Quantum Grav. 21, pp. S403-S408, 2004.

[5] S. Goßler, The suspension systems of the interferometric gravitational-wave detector GEO 600, PhD thesis, Chapter 4, pp. 95-118, and Chapter 5, pp. 142-143, University of Hannover, 2004.

[6] E. J. Elliffe et al, Hydroxide-catalysis bonding for stable optical systems, Class. Quantum Grav. 22, pp. S257-S267, 2005.

[7] G. I. Gonzalez and P. R. Saulson, Brownian motion of a mass suspended by an anelastic wire, J. Acoust. Soc. Am. 96(1), pp. 207-212, 1994.

[8] J. R. Smith et al, Linear projection of technical noise for interferometric gravitational-wave detectors, Class. Quantum Grav. 23, pp. 527-537, 2006.

[9] I. A. Bilenko and S. L. Lourie, Measurements of effective noise temperature in fused silica fiber violin modes, Physics Letters A 305, pp. 31-35, 2002.

[10] A. Yu. Ageev, I. A. Bilenko, V. B. Braginsky and S. P. Vyatchanin, Measurement of excess noise in the suspension fiber for a gravitational wave detector, Physics Letters A 227, pp. 159-164, 1997.

[11] I. A. Bilenko, V. B. Braginsky and N. Yu. Markova, Thermal and excess noise in suspension fibres, Class. Quant. Grav. 19, pp. 2035-2038, 2002.

[12] G. Cagnoli et al, Mechanical shot noise induced by creep in suspension devices, Physics Letters A 237, pp. 21-27, 1997 .

[13] M. Beccaria et al, The creep problem in the VIRGO suspensions: a possible solution using Maraging steel, Nucl. Instrum. Meth. A 404, pp. 455-469, 1998.

[14] G. Cagnoli et al, Silica suspension and coating developments for Advanced LIGO, Journal of Physics: Conference Series 32, pp. 386-392, 2006.

[15] Advanced LIGO Team, Advanced LIGO reference design, LIGO-M060056, 2006. http://www.ligo.caltech.edu/docs/M/M060056-08/M060056-08.pdf

[16] R. Flaminio et al (for the VIRGO Collaboration), Advanced Virgo white paper, Virgo Note VIRNOT-DIR-1390-304, 2005. http://wwwcascina.virgo.infn.it/advirgo/docs/whitepaper.pdf

[17] A. M. Gretarsson, P. R. Saulson, Monitoring the thermal and nonthermal excitation of fibers, Review of Scientific Instruments 76, pp. 054502-1-054502-11, 2005.

[18] I. A. Bilenko, N. Yu. Lyaskovskaya, The investigation of thermal and non-thermal noises in fused silica fibers for Advanced LIGO suspension, Physics Letters A 339, pp. 181-187, 2005.

[19] S. Goßler et al, Damping and tuning of the fibre violin modes in monolithic silica suspensions, Class. Quantum Grav. 21, pp. S923-S933, 2004.

[20] R. Balasubramanian, H. Grote, I. S. Heng, M. Hewitson, H. Lück, J. R. Smith, K. A. Strain, H. Ward and B. Willke, Results from the first burst hardware injections performed on GEO 600, Class. Quant. Grav. 22, pp. 3015-3028, 2005.

[21] I. S. Heng, R. Balasubramanian, B. S. Sathyaprakash and B. F. Schutz, First steps towards characterizing the hierarchical algorithm for curves and ridges pipeline, Class. Quant. Grav. 21, pp. S821-S826, 2004.

[22] J. R. Pugh, Experiments on and relating to the detection of gravitational radiation, $\mathrm{PhD}$ thesis, Chapter 3, University of Glasgow, 1976. 